\title{
A simulation model for bioterrorism preparedness in urban areas
}

\author{
W. C. Degnan ${ }^{1}$, E. I. Kaisar ${ }^{1} \&$ J. W. Schokkin ${ }^{2}$ \\ ${ }^{1}$ Department of Civil Engineering, Florida Atlantic University, USA \\ ${ }^{2}$ Department of Civil Engineering, University of Twente, The Netherlands
}

\begin{abstract}
With the ever increased security throughout the world's countries, terrorists have begun to find new ways to inflict fear upon the masses. One such method is the use of a bioterrorist attack, otherwise known as bioterrorism. Bioterrorism is the intentional release of biological agents, such as bacteria, viruses, and toxins. These agents are able to be spread through the air, the water supply, or even the food supply. One feared agent that could be released is the smallpox virus. This is a potentially deadly disease that can take several days to detect. The reason that a bioterrorist attack would be successful throughout the world is because it is virtually undetectable. There is no technology today that can determine if someone is carrying a biological agent. A well devised attack plan is the only way to counter a bioterrorist attack. This is done by the use of computer simulation software. Traffic simulation software can be used to map and plan evacuation routes in a large city (urban area). The spread pattern of a released agent can also be determined with the help of software. An important aspect to simulate this scenario would be the influx of people entering the hospitals and emergency centers. A good plan needs to be devised to determine where to put the sick people getting treatment, and where to put the people who could be potentially sick but are not showing any symptoms. Simulation software would allow agencies to develop a plan beforehand so that in the event of an attack they will be ready and mass casualties would be avoided.
\end{abstract}

Keywords: traffic simulation, urban areas, bioterrorism, planning, response, emergency centers. 


\section{Introduction}

The unstable state of foreign relations that exist worldwide due to prior and looming terrorist attacks has changed the face of event preparation. The severity of possible attacks immediately gains the attention of those in charge of catastrophic event preparedness. This study presents a simulation-based process to analyze a metropolitan area, such as Baltimore City, and diagnose possible conflict areas in the time of a response situation. The infected area for this case study is contained inside the I-695 beltway that encompasses the city as well as the Baltimore-Washington International Airport. The network is comprised of 631 traffic analysis zones, inside Baltimore County and Baltimore city boundaries. The population that we will be dealing with inside the network boundaries is approximately 951,000 people. The macro/meso level simulation platform will use the existing road network for arterial routes to emergency centers. An array of smaller more detailed networks, microscopic simulation, will deal specifically with the corridors and routes that lead directly from major highways to emergency centers. Most of the intersections will be controlled with traffic signals or stop/yield signs. The traffic signal control will contain actual timings obtained from the Baltimore Metropolitan Council (BMC). Emergency centers will be predetermined for treatment, isolation and/or quarantine. The emergency centers are determined by the command control, and are located around the network to service each Transportation Analysis Zones (TAZ). The emergency centers that will be used in the case study are hospitals in Baltimore County and Baltimore City metropolitan area.

\section{Literature review}

The review of literature was performed in two critical areas. The first area that was studied dealt with bioterrorism and the spread of disease through a network. Most of the studies in this area concentrated on the spread of disease through contact rates, contact locations and the timeline of signs and symptoms. Much of the bioterrorist models were structured to observe contact patterns inside a network [10]. A common contact mixing scheme inside a typical network can be seen in Figure 1. These models found schools, neighborhoods and social gatherings to be breeding grounds for the spread of disease $[2,10]$. In studies based on smallpox $[2,6,9,10,12]$, it was very important to identify the nature of the disease because it has different characteristics than most biological weapons.

This is what creates the very high risk of infection and/or death because the signs and symptoms do not appear for approximately 12 days. The timeline is well defined by Chen et al. [6] and Longini et al. [12], incubation, prodrome (early signs) and fulminant (late stages) can all be seen in Figure 2.

- Incubation- 12 to 14 days, no symptoms

- Prodrome (early-symptoms)- 2 to 4 days, fever, aches

- Fulminant (late-symptoms)- 7 to 14 days, rash

Other studies by Bozzette et al. [2] and Eubank et al. [8] study the impacts of vaccination both prior and during the spread of disease. It was almost 
unanimous that healthcare workers and officials should most definitely be vaccinated prior to an event. This allows them to work uninhibitedly and respond to those in need of treatment. Network-wide vaccinations are found unproductive and should only be implemented if the risk of an attack becomes so prevalent that it is near demanded. These papers provided insight as to where and how many vaccination/emergency centers are necessary. Aside from just having emergency centers it was established that the ability to have them on stand-by and to have the capacity to open or close them as needed due to surge capacity is a capability unexplored. Response studies, Ferguson et al. [9] and Glasser et al. [10], both make it clear that effective targeting and containment of know infected areas can greatly reduce the spread and infection of disease. Thus, it reduces the overall demand for treatment at emergency centers, lowering travel delays.

Perhaps the most important idea that was gathered from the bioterrorism review was that analysis of the social network's structure should be done in enough detail to understand how and where it interacts (Demographics).

The second critical area that was explored through the review of literature was in simulation. Simulations were reviewed in evacuation studies. There is

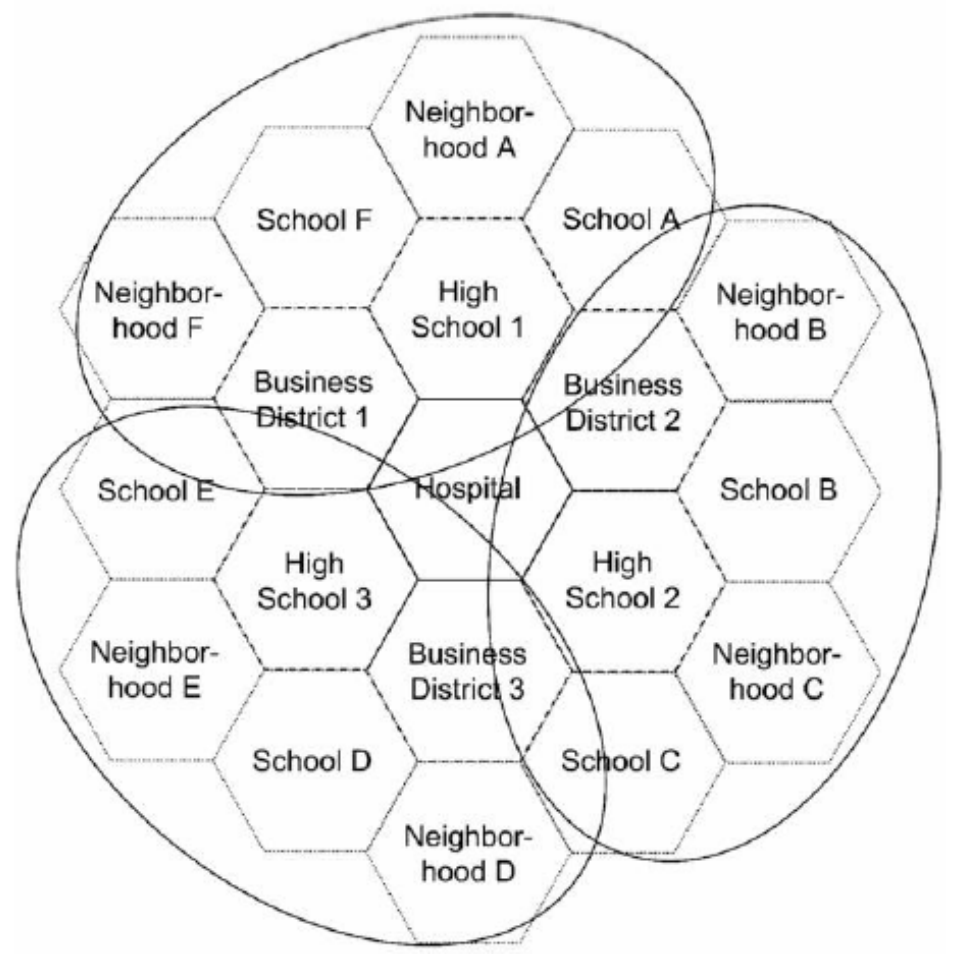

Figure 1: Disease contact mixing scheme (Glasser et al. [10]). 


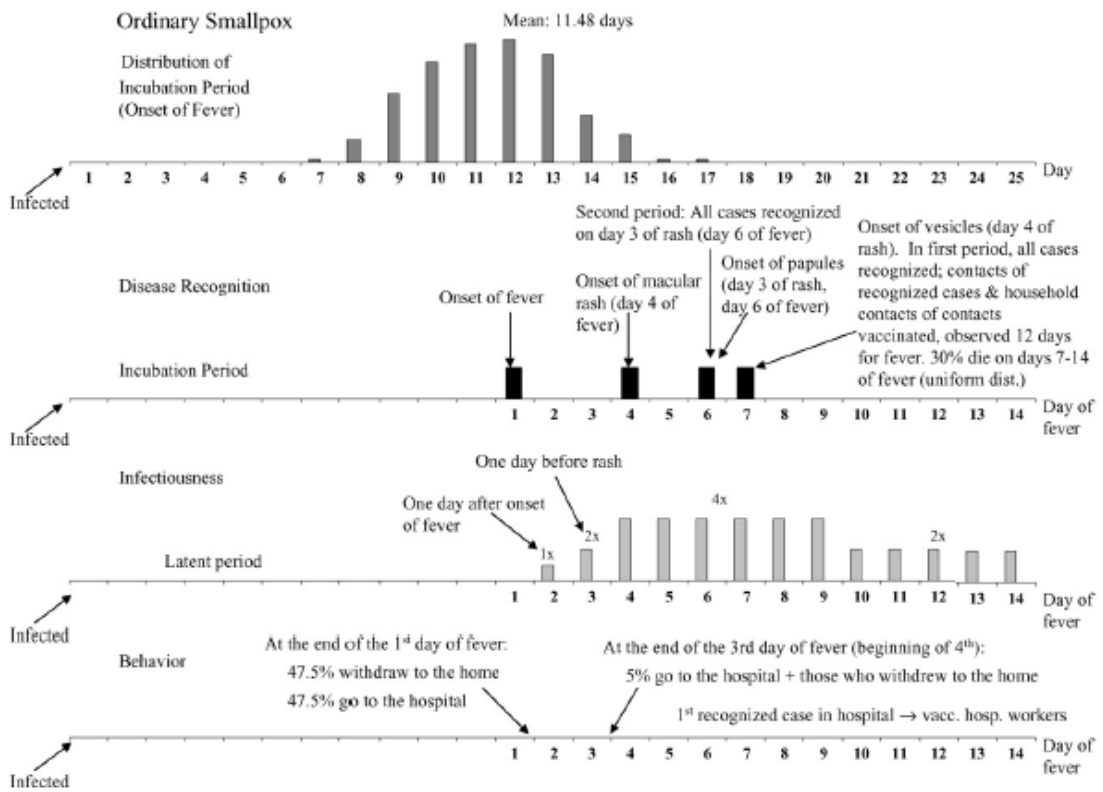

Figure 2: $\quad$ Onset of disease timeline (Longini [17]).

very little research done regarding simulation in the field of catastrophic response because of the lack of real-life examples, case studies and the difficulty to reproduce accurate representations. Therefore, three levels of simulation were studied to better understand the capabilities and drawback of each to determine the best method to enhance catastrophic response. The first level involved macro/mesoscopic simulations. This area is very important in our case study because it deals with masses of people (flows) and their ability to reach a variety of locations within the boundaries of the network. This level of study is dealing with capacities and flow-densities around and through the network $[11,13,15]$. It was pertinent according to Liu et al. [11], that there be several stages in place for a successful evacuation; Plan, Contingency, Simulation and Review. To delve into the finer details of our case study it was necessary to review microsimulation which handles the traffic movements and now driver behavior. A study performed by Sterzin et al. [16] details the capabilities of different microsimulation research programs. Microsimulation requires very fine details in order to obtain valid and accurate results. Some of the details outlined in research include but are not limited to, turning movements, free flow speed and signal control timings. Accuracy is lost in the area of driver behavior. Driver behavior is especially hard to model in the event of a crisis due to the high level of uncertainty that drivers will, follow authoritative guidelines, obey signal controls and act in an orderly fashion. Erratic behavior can be monitored in during real-time situations and those actions can be simulated through the API interface in Aimsun. The Baltimore Case Study makes great use of this feature, 
as it enhances the ability to account for driver behavior, which is currently the greatest unknown.

The new progression for simulations is seen in research performed by Burghout et al. [4], where they combined levels of scope for a more complete diagnosis of the simulation. The meso and micro levels of simulation were meshed together into a hybrid type of network. This allows for a more complete diagnosis of the network, monitoring small network that lead to emergency centers within the beltway, while still monitoring the larger surrounding flows and queuing schemes. This hybrid technique allows a more general type of network (meso) to be analyzed in a way it wouldn't be capable of prior. While the highly detailed micro networks can retract some of the painful detailing while still maintaining accuracy.

\section{Simulation network}

Presented in Figure 3, is the system framework of the integrated catastrophic emergency response plan. The red square outlines the portion of a bioterrorist attack response, in which simulation will provide insight and guidance during planning and response of emergency personal and centers. A response will consists of multiple origin-destination (O/D) matrices which will have the capacity to be opened, closed and/or rerouted based on the situational awareness and surge capacity information from command control. Due to the nature of the disease in the bioterrorist attack (delayed onset of signs and symptoms) the traffic management perspective will have the capability to evolve alongside the changes in demand for emergency centers. Coding scripts will be modeled in order to accurately visualize and manage the driver behavioral aspects of real-life scenarios or simulations. Due to the uncertain nature of human behavior in emergency situations it is important to have the ability to manage abnormal actions taken by drivers to arrive at emergency centers. The system can be broken down into steps to help analyze the processes that will be working inside the case study.

1) Modeling of current network to reproduce mixing conditions of general population due to the contact based spreading of disease.

a. Using demographics and trip distribution

b. Mesoscopic modeling of traffic flow and trips, through speeddensity relationship and queuing theories.

2) Once command control establishes an Emergency response scenario, the emergency enters per TAZ are assigned.

a. Microscopic simulation regarding travel to emergency centers.

b. Network allows visualization and situational awareness during real-time response in regard to fluctuations in demand and surge capacity

3) Due to the nature of the disease (delayed onset of signs and symptoms) rates of demand will be skewed through stochastic simulations. Based on day of simulation, actual contact and worried well rates. 


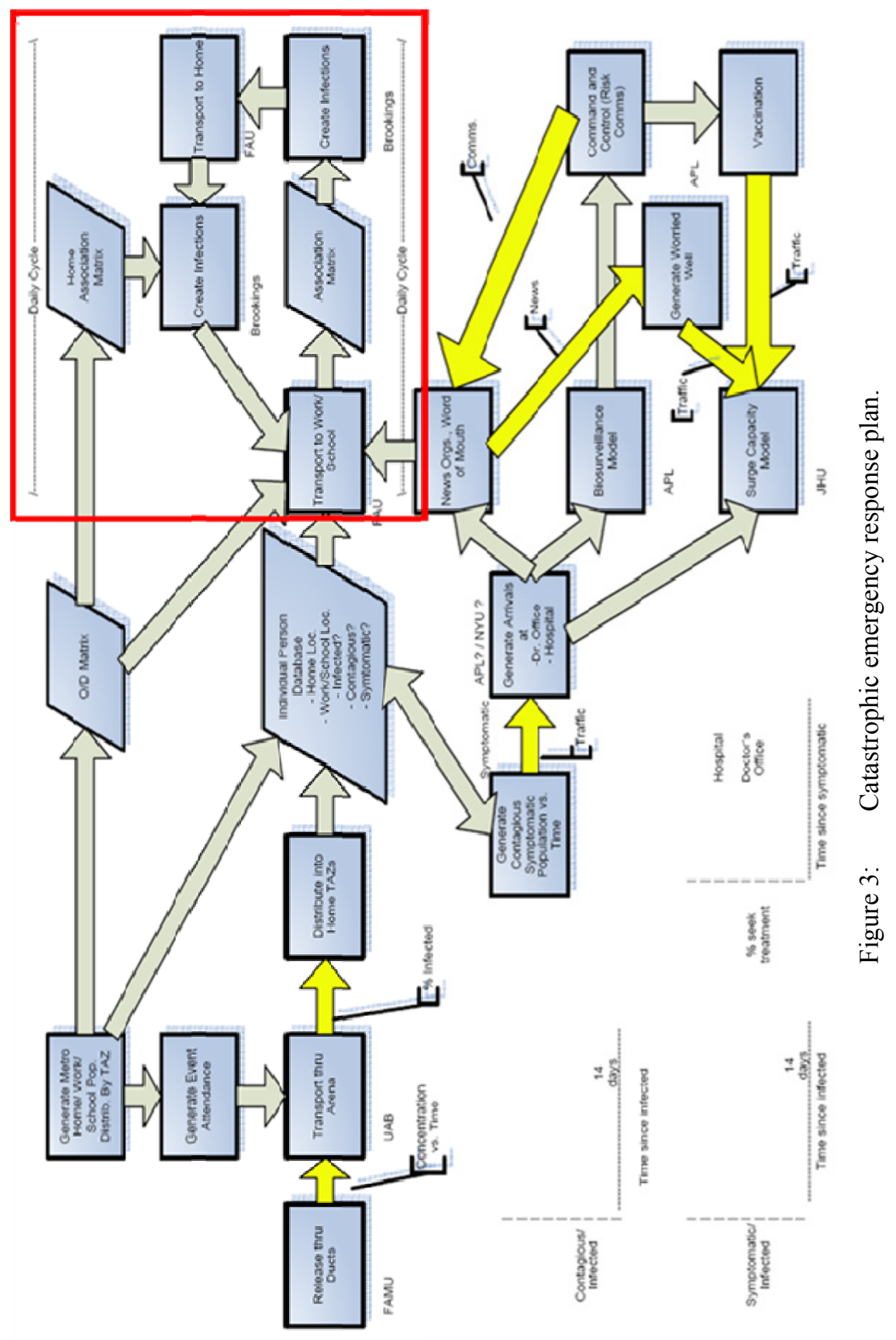


a. Through AIMSUN's ability to use its Application Programming Interface (API) script written in $\mathrm{C}++$. These extensions are used to dynamically modify the current simulation by changing, for instance, driver parameters, control timing, implementing powerful traffic management actions...

4) The mesoscopic and microscopic networks will be intertwined in a hybrid meso-micro traffic simulation.

a. Allows analysis of specific areas of interest, while monitoring the flows of a greater surrounding network

\subsection{Optimization}

Optimization of the network will become critical to employ the most efficient response strategy. The optimization will be two-fold. First, the designated emergency center routing strategy and secondly the optimal command control strategy that allows access to the simulation interface during real-time simulation to account for surge capacity in the form of traffic delays of back-up times at the centers. Optimization becomes extremely important during real-time operations because it can efficiently identify the number of centers that are required to be in operation to handle the demand of traffic en-route to obtain treatment. It is also a tool to account for unexpected accidents, roadway damage and erratic driver behavior.

\subsection{System application}

This section presents the application of the proposed catastrophic event response plan for Baltimore M.D. This case study can be used as a training tool assisting users in manipulation of a catastrophic event scenario. Analyzing events such as accidents, lane closures, abnormal driver behavior and heavy emergency treatment demand. These occurrences can be accounted for by altering traffic assignments through new $\mathrm{O} / \mathrm{D}$ matrices and routing techniques.

The bioterrorist attack scenario takes place at a concert area in downtown Baltimore City, Maryland. The crowd at the concert is said to be in their late teens. Based on demographic research inside each TAZ, a distribution of attendees is created based on TAZ origin. The attack will take place through the air duct network of the arena. The attendees of the show will be unaware of the release of smallpox and will head home after the show. Normal daily operations regarding trip distribution throughout the network will be monitored for the next 12 days (based on smallpox model for incubation) during morning peak hour times. After approximately 12 days the first signs and symptoms begin to arise and emergency centers will be implemented throughout the network as necessary.

\section{Simulation results and analysis}

In this chapter an overview of the results acquired from the micro-simulation model is given. One of the zones in the downtown area where the attack takes 
place was modeled to obtain measure of performance therefore we can adjust the whole model accordingly. The delay time, density, flow, harmonic speed, speed, stop time, stops, total distance traveled, total travel time, evacuation time and lost vehicles from all the different replications are listed. Also average values are calculated and listed for the different evacuation strategy. For each strategy the total travel time is illustrated in a graph. A summary of the simulation results regarding the measurement of effectiveness (MOEs) described in section listed in Table 1. The results from the pair comparison of the main strategies are illustrated in Figure 4. It can be concluded that the Reference strategy is not effective at all. Regarding the evacuation time the scores for the rest of the strategies are very close together. The results of the Nearest Exit 1 strategy however, are very unlikely compared to the results provided by the evacuation calculator. The short evacuation time is likely caused by the great loss of vehicles. The Nearest Exit 2 strategy, in which all the six exits are available instead of four, produces more likely results. The Management strategy produces the best evacuation time results. It can be concluded that the Management and both Staged strategies show promising results. It would be difficult to implement a staged evacuation in reality, because in a life threatening situation it would be almost impossible to delay people entering the network, therefore the Management strategy is the most effective.

Table 1: $\quad$ Final simulation results, MOEs.

\begin{tabular}{|c|c|c|c|c|c|c|c|}
\hline \multicolumn{8}{|c|}{ Final Simulation Result } \\
\hline & \multicolumn{2}{|c|}{$\begin{array}{l}\text { Total Evacuation } \\
\text { Time }(\text { h) }\end{array}$} & \multicolumn{2}{|c|}{ Total Travel Time (h) } & \multicolumn{2}{|c|}{$\begin{array}{l}\text { Total Distance } \\
\text { Traveled }(\mathrm{km})\end{array}$} & \multirow{2}{*}{$\begin{array}{l}\begin{array}{l}\text { Lost } \\
\text { Vehicles \# }\end{array} \\
\text { Mean Value }\end{array}$} \\
\hline Strategy & Mean Value & Std. Dev. & Mean Value & Std. Dev. & Mean Value & Std. Dev. & \\
\hline Nearest Exit 1 & 4.83 & 0.14 & 25768.17 & 1092.63 & 70855.67 & 547.10 & 46279 \\
\hline Nearest Exit 2 & 5.50 & 1.39 & 20489.67 & 2754.48 & 70304.83 & 4423.17 & 29959 \\
\hline Reference & 10.58 & 1.38 & 38873.30 & 7622.39 & 79975.73 & 5426.19 & 36293 \\
\hline Management & 5.08 & 0.63 & 21334.70 & 862.13 & 69844.97 & 1086.38 & 29153 \\
\hline Staged 1 & 6.00 & 0.43 & 16809.43 & 496.58 & 78004.67 & 244.47 & 27318 \\
\hline Staged 2 & 5.58 & 0.29 & 16859.93 & 42.87 & 77886.37 & 1071.98 & 28390 \\
\hline Contra Flow & 5.33 & 0.52 & 19699.83 & 2642.90 & 66737.03 & 6135.93 & 30977 \\
\hline Parked Cars & 5.50 & 0.25 & 24705.13 & 846.07 & 68746.27 & 1089.03 & 30336 \\
\hline Signal Control 1 & 7.58 & 0.14 & 22735.60 & 2146.21 & 68136.10 & 3821.18 & 32133 \\
\hline Signal Control 2 & 4.67 & 0.29 & 23255.73 & 1358.51 & 71266.63 & 597.68 & 32002 \\
\hline No Control & 3.00 & 0.00 & 14070.43 & 669.75 & 72602.27 & 1102.52 & 30056 \\
\hline No Control Fixed & 3.00 & 0.00 & 13332.5 & 480.1 & 63551.4 & 1514.9 & 29581 \\
\hline $\begin{array}{l}\text { No Control Fixed } \\
\text { Best Entrance }\end{array}$ & 6.75 & 0.00 & 3657.1 & 48.5 & 46249.2 & 154.0 & 11785 \\
\hline
\end{tabular}

Two complementary sub-strategies are finally carried out to gain more insight into the limitations of the chosen C-Logit route choice model. Although only converging and some diverging traffic flows should appear because trips are distributed regarding the shortest path. A closer look to the 'Interactive Simulation' in micro/meso simulation platform indicates that many diverging and crossing traffic flows appear. This is probably caused by the C-Logit route choice model which calculated new shortest routes every 15 minutes. The Fixed route choice model is static, calculating only once the shortest routes at the beginning of the simulation, and uses only one shortest route for each OD-pair. 


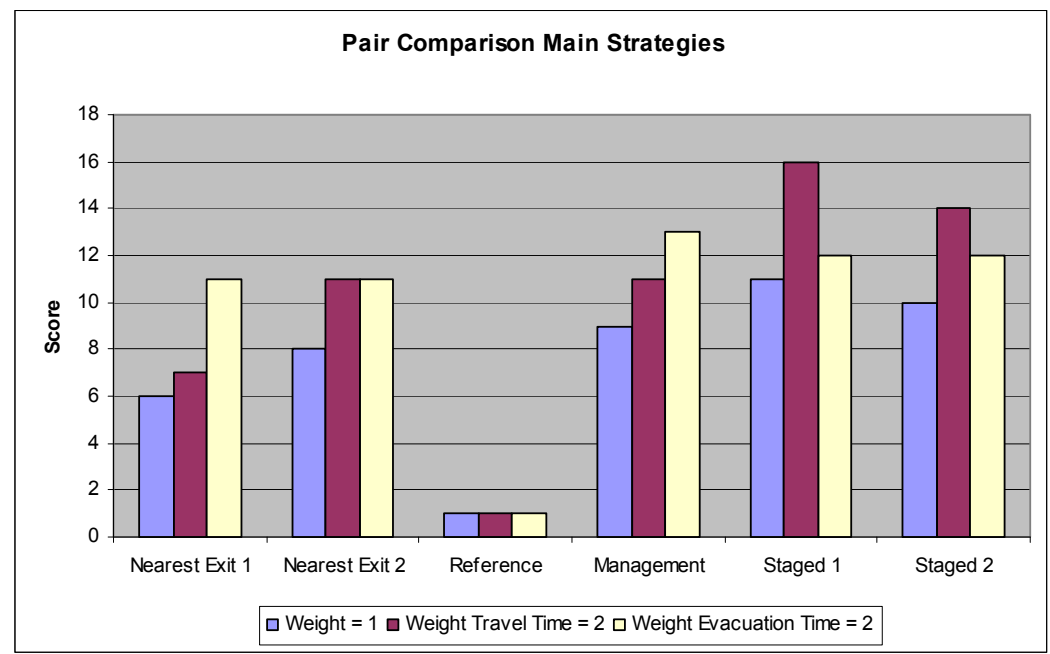

Figure 4: $\quad$ Pair comparison main strategies.

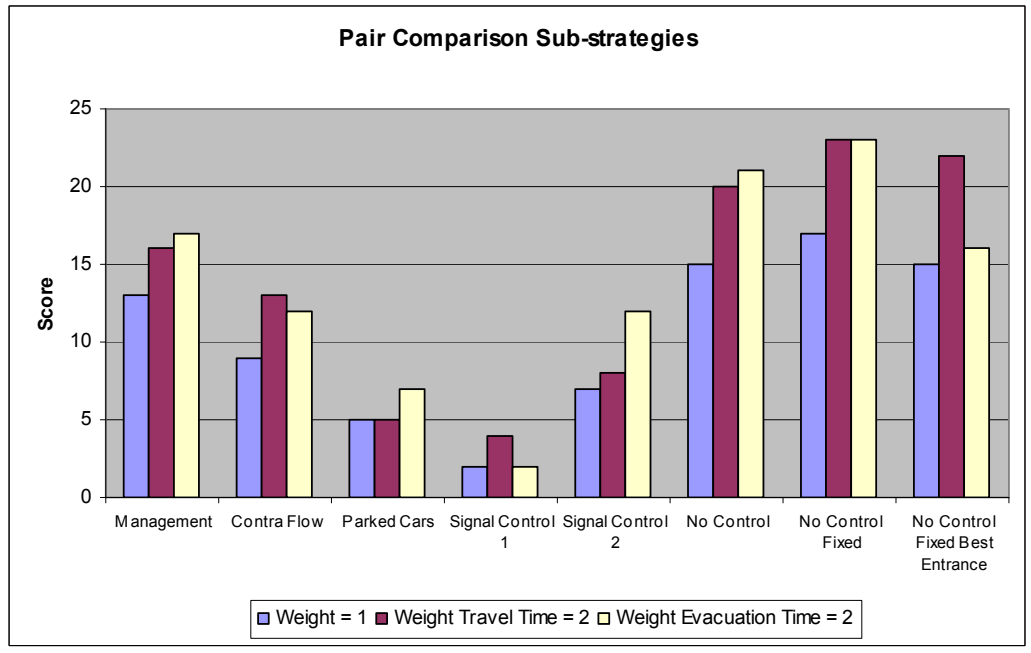

Figure 5: $\quad$ Pair comparison sub-strategies.

The No Control Fixed strategy shows better results, but not significant. Also the 'Interactive Simulation' still indicates many crossing and diverging traffic flows. Initially, an equal and fixed percentage of vehicles are distributed over the origin centroids connectors. As a result of this some vehicles need to make a detour to finally reach their desired shortest route. To avoid this problem only the best origin centroid connectors are used in the final strategy; No Control Fixed Best Entrance. 
This strategy shows a significant decrease in total travel time, total distance traveled and lost vehicles. However, the total evacuation time is more than doubled up to 6.75 hour. A promising result however is that this delay is almost completely caused by a fraction of the evacuation traffic, see figure 5 .

\section{Conclusion}

This paper has presented a case study regarding catastrophic event planning and response for the Baltimore M.D. metropolitan area. The proposed simulation and optimization allows for effectiveness in monitoring mixing of the population and management of treatment centers through routing of real-time behaviors and demands. Two levels of simulation meshed into an optimal hybrid simulation along side, $\mathrm{O} / \mathrm{D}$ matrices, optimal signal timing and real-time routing manipulation are all part of the critical response plan.

For the downtown area microsimulation initially four evacuation strategies are developed (Nearest Exit, Reference, Management, Staged) resulting in 12 sub-strategies. The trip end calculation found that the evacuation of the area will take at least 2 hours and 52 minutes. The evacuation calculator calculated 17 hours and 40 minutes, 3 hours and 20 minutes, and 4 hours and 20 minutes of evacuation time for the nearest exit, management and reference strategy respectively.

Finally the different strategies/sub-strategies are implemented in the developed simulation model. This microscopic dynamic model contains only the major arterials of the area, 22 origin zones, 12 exit roads leading to 6 safe destination zones, a 9:00 AM traffic demand and a fixed PM-peak control plan. Parameters for route choice and human behavior are set to emergency evacuation driver behavior. Due to the unavailability of emergency evacuation field data and sufficient time, quantitative calibration of the model was not possible. However with the result produced by the model the different strategies can be compared, which is sufficient regarding the goal of this research.

Pair comparison of the results from the different strategies regarding the three Measures of Effectiveness (evacuation time, total travel time and lost vehicles) concluded the management strategy as the most effective. This strategy results in an evacuation time of 5.08 hours with a total travel time of 21335 hours. The staged evacuation time shows promising results, pertaining to the low total travel time of 16809 hours and its ability to evacuate the people nearest to the disaster first.

These types of simulations will be conducted throughout the network at each of the centroids which contain multiple traffic analysis zones. These microsimulation networks are meshed with the mesoscopic simulation of the normal daily traffic operation along the beltway and major traffic infrastructures throughout.

\section{References}

[1] Arnon, Stephen S.; Robert Schechter; Thomas V. Inglesby; et al. (2004) Botulinum Toxin as a Biological Weapon: Medical and Public Health Management 
[2] Bozzette, Samuel A. M.D., Ph.D., Rob Boer, Ph.D., Vibha Bhatnagar, M.D., M.P.H., et al.(January 2003). A Model for a Smallpox-Vaccination Policy

[3] Buckeridge, David L.,, , Howard Burkomc, Murray Campbelld, William R. Hogane, Andrew W. Mooref, (November 2004) Algorithms for rapid outbreak detection: a research synthesis

[4] Burghout, Wilco, Haris N. Koutsopoulos, Ingmar Andréasson (2005) Hybrid Mesoscopic-Microscopic Traffic Simulation

[5] Carley, Kathleen M. Neal Altman, Boris Kaminsky, Démian Nave and Alex Yahja. (January 2004). BioWar: A City-Scale Multi-Agent Network Model of Weaponized Biological Attacks

[6] Chen, Li-Chiou, Boris Kaminsky, Tiffany Tummino, Kathleen M. Carley, Elizabeth Casman, Douglas Fridsma, and Alex Yahja, (2004) Aligning Simulation Models of Smallpox Outbreaks

[7] Eubank, Stephen. (2002) Scalable, Efficient Epidemiological Simulation

[8] Eubank, Stephen Hasan Guclu, V. S. Anil Kumar, Madhav V. Marathe, Aravind Srinivasan, Zolta'n Toroczkai \& Nan Wang. (May 2004) Modeling disease outbreaks in realistic urban social networks

[9] Ferguson, Neil M., Matt J. Keeling2, W. John Edmunds3, Raymond Gani4, Bryan T. Grenfell5, Roy M. Anderson1 \& Steve Leach4. (October 2003) Planning for smallpox outbreaks

[10] Glasser, John W., Stanley O. Foster, J. Donald Millar, and J. Michael Lane (2008) Evaluating Public Health Responses to Reintroduced Smallpox via Dynamic, Socially Structured, and Spatially Distributed Metapopulation Models

[11] Liu, Yue; Gang-Len Chang, Ying Liu, Xiaorong Lai. A Corridor-Based Emergency Evacuation System for Washington D.C.: System Development and Case Study

[12] Longini, Ira M. Jr., M. Elizabeth Halloran, Azhar Nizam, Yang Yang, Shufu Xu, Donald S. Burke, Derek A.T. Cummings Joshua M. Epstein. (March 2006) Containing a large bioterrorist smallpox attack: a computer simulation approach

[13] McGhee, Catherine C., P.E., Matthew C. Grimes. (2007). An Operational Analysis of the Hampton Roads Hurricane Evacuation Traffic Control Plan

[14] Moriarty, Kevin D., Daiheng Ni, and John Collura. (November 2006). Modeling Traffic Flow under Emergency Evacuation Situations: Current Practice and Future Directions

[15] Southworth, Frank. (March 1991). Regional Evacuation Modeling: A Stateof-the-Art Review.

[16] Sterzin, Emily D., Tomer Toledo, Moshe E. Ben-Akiva. (November 2004) Influencing Factors in Microscopic Traffic Simulation

[17] Longini, I., Halloran E., Nizam A., Yang Y., Xu S., Burke D., Cummings D., and Epstein J., Containing a large bioterrorist smallpox attack: a computer simulation approach," International Journal of Infectious Diseases (2007) 11, 98-108. 